\title{
Praticando a teoria no ensino de IHC: dinamizando aulas teóricas com o uso de atividades práticas
}

\author{
Milene Selbach Silveira \\ Escola Politécnica \\ Pontifícia Universidade Católica do Rio Grande do Sul \\ Porto Alegre - RS - Brasil \\ milene.silveira@pucrs.br
}

\section{RESUMO}

O dinâmico mercado atual necessita de profissionais com competências além daquelas relacionadas a sua área de atuação. Trabalhar a colaboração, a solução de problemas, a curiosidade, a imaginação e o pensamento crítico são fundamentais neste contexto. Considerando-se este cenário, serão apresentadas atividades práticas desenvolvidas durante uma disciplina de IHC, a fim de promover não apenas o desenvolvimento destas competências, mas o engajamento e o protagonismo do aluno.

\section{Palavras chave}

atividades práticas, dinâmicas de grupo, ensino de IHC

\section{INTRODUÇÃO}

Atualmente, formar profissionais para atuação em um mercado dinâmico, é um grande desafio para os professores em geral. Além dos conteúdos específicos de cada área do conhecimento, é necessário que estes profissionais em formação desenvolvam competências como a colaboração, a solução de problemas, a curiosidade e a imaginação e o pensamento crítico, dentre outras [6].

A fim de se desenvolver estas competências e, também, motivar os alunos a se envolverem mais com o seu aprendizado, diferentes estratégias metodológicas têm sido experimentadas. Ouve-se muito falar em metodologias (inov)ativas [2][5][6][8], as quais têm, como princípios essenciais, o protagonismo do aluno, a açãoreflexão e a colaboração [6]. Neste rol de metodologias, encontram-se a sala de aula invertida, a avaliação por pares, a aprendizagem baseada em problemas e em projetos, os jogos e a gamificação, dentre outros.

Permission to reproduce or distribute, in whole or in part, material extracted from this work, verbatim, adapted or remixed, as well as the creation or production from the content of such work, is granted without fee for non-commercial use, provided that the original work is properly credited.

IHC 2019 Workshop sobre Educação em IHC (WEIHC), Outubro 2125, 2019, Vitória, Brasil. In Anais Estendidos do XVIII Simpósio Brasileiro sobre Fatores Humanos em Sistemas Computacionais. Porto Alegre: SBC.

(C) 2019 by the author(s), in accordance with the terms of the Creative Commons Attribution-NonCommercial 4.0 International Public License (CC BY-NC 4.0).
Considerando-se a área de Computação, o movimento de computação desplugada (unplugged Computer Science) [3][4] se destaca neste sentido, ao estimular o aprender fazendo, a diversão, a inclusão e a cooperação, princípios estes que promovem um maior envolvimento dos participantes. Para a área de Interação Humano-Computador (IHC), especificamente, encontram-se várias iniciativas neste contexto desplugado [1][3][7][11].

Neste cenário, serão apresentadas atividades variadas que buscam dinamizar aulas teóricas de IHC. Apesar de já terem sido utilizadas em diferentes disciplinas da área, neste artigo elas serão apresentadas no contexto de uma disciplina de IHC específica, do curso de Bacharelado em Engenharia de Software da Pontifícia Universidade Católica do Rio Grande do Sul (PUCRS).

As seções a seguir descrevem em maior detalhe a disciplina em si e uma série de exemplos de atividades que têm sido ministradas na disciplina em questão, além de considerações finais e das referências utilizadas para sua construção.

\section{A DISCIPLINA}

Considerando o curso de Engenharia de Software da PUCRS, a área de IHC contempla duas disciplinas: Fundamentos de IHC, disciplina teórica de 4 créditos, de cunho obrigatório, oferecida no $3^{\circ}$ semestre do curso, e Design de Interação, disciplina prática, de 2 créditos, também obrigatória, oferecida no $4^{\circ}$ semestre. Neste artigo, o foco está na primeira disciplina: Fundamentos de IHC.

Esta disciplina tem como objetivo introduzir a área de IHC, seus principais conceitos e teorias de base, bem como diferentes métodos de avaliação de qualidade de uso e os princípios do processo de design de interação. Na Tabela 1 é visto um resumo dos principais tópicos trabalhados na disciplina, organizados de acordo com cada uma de suas unidades programáticas.

A fim de dinamizar o processo de ensino e de aprendizagem, variadas atividades práticas são realizadas ao longo da disciplina, conforme será discutido na próxima seção. 
Tabela 1: Principais tópicos trabalhados na disciplina

\begin{tabular}{l|l}
\hline \multicolumn{1}{c|}{ Unidade } & \multicolumn{1}{c}{ Tópicos Principais } \\
\hline $\begin{array}{l}\text { Contexto da Área de } \\
\text { IHC }\end{array}$ & $\begin{array}{l}\text { objetivos, multidisciplinaridade, } \\
\text { evolução, aspectos éticos, mercado } \\
\text { de trabalho }\end{array}$ \\
\hline Princípios Básicos & $\begin{array}{l}\text { conceitos básicos, qualidade de uso, } \\
\text { aspectos cognitivos, culturais e } \\
\text { sociais, abordagens teóricas, } \\
\text { processos de design }\end{array}$ \\
\hline Avaliação em IHC & planejamento, métodos de avaliação \\
\hline $\begin{array}{l}\text { Princípios de Design } \\
\text { de Interação }\end{array}$ & elicitação e análise de dados \\
\hline
\end{tabular}

\section{AS PRÁTICAS}

Conforme apresentado na Tabela 1, são quatro as unidades programáticas desta disciplina específica. As duas primeiras são inerentemente teóricas, para as quais se começou a buscar por atividades que promovessem um maior engajamento e participação dos alunos.

$\mathrm{Na}$ parte inferior da Figura 1, temos indicados diferentes momentos da disciplina em que as atividades a serem aqui apresentadas ocorrem, destacando-se que esta imagem é ilustrativa, não correspondendo efetivamente a carga horária destinada a cada Unidade.

As atividades serão discutidas nas próximas subseções, organizadas de acordo com as diferentes unidades em que os conteúdos relacionados são trabalhados.

\subsection{Atividades: Contexto da Área de IHC}

Durante a primeira unidade, referente ao contexto da área de IHC, destacam-se as seguintes atividades:

- Evolução: para discussão da evolução de IHC, a turma é dividida em grupos, para construção de linhas do tempo. Em um primeiro momento, cada grupo se concentra em procurar informações sobre uma década específica e, em um segundo momento, toda a turma compartilha as informações coletadas para criação das linhas do tempo (uma por grupo). Após a finalização da tarefa, os alunos são estimulados a analisar as linhas criadas por todos os grupos e, na aula seguinte, uma discussão sobre esta evolução é realizada.

- Questões éticas: após apresentação e discussão sobre questões éticas em geral e em IHC, são disponibilizados artigos curtos sobre diferentes assuntos relacionados ao tema (dark patterns, GDPR, desafios éticos de design, etc). Primeiro os alunos leem o artigo de seu interesse e depois os discutem em grupos com colegas que analisaram artigos do mesmo assunto. Por fim, há uma discussão geral em aula, com sínteses, pelos diferentes grupos, do que foi por eles discutido.
- Mercado: na aula que trata sobre o perfil do profissional de IHC/UX e o mercado de trabalho, os alunos analisam, individualmente, pesquisas que mostram o perfil do profissional da área e, em grupos, discutem pontos que lhe chamaram a atenção e elencam e analisam um subconjunto das atividades/ metodologias, citadas nas pesquisas, que lhes chamou a atenção.

\subsection{Atividades: Princípios Básicos}

Durante a segunda unidade, referente aos princípios básicos de IHC, destacam-se as seguintes atividades:

- Aspectos cognitivos: na aula sobre aspectos cognitivos, diferentes dinâmicas são realizadas, com "mini jogos" relacionados a cada um dos aspectos cognitivos, como atenção, memória, etc. A cada nova atividade um subgrupo de alunos - em diferentes configurações (duplas, trios, etc) - vai para "frente" da sala e executa a atividade indicada (por exemplo: jogo de memória em que - a partir de um tópico selecionado pela turma cada participante (de um grupo de 6) deve citar as palavras que os anteriores falaram, sem pular ou repetir nenhuma). Os resultados são discutidos a cada jogo realizado.

- Engenharia cognitiva: para discussão específica da Teoria da Ação, os alunos, após refletirem sobre a aplicação da teoria (e travessia dos golfos de execução e avaliação) a diferentes cenários, devem criar seus próprios cenários e compartilhar com a turma (que é convidada a executá-los).

- Engenharia semiótica: para discutir o conceito de comunicabilidade, é realizada uma dinâmica ao estilo do jogo Imagem\&Ação®, na qual cada grupo deve projetar um ícone (de acordo com a funcionalidade - não usual recebida) e os demais grupos devem adivinhar qual a funcionalidade representada (discutindo as questões de baixa e alta comunicabilidade dos mesmos além das dificuldades de representações associadas). Além disto, é feita a realização de exercícios diversificados sobre a mensagem do designer e a análise de signos (metalinguísticos, estáticos e dinâmicos) em diferentes aplicativos (escolhidos pelos alunos dentre os que eles têm disponíveis em seus smartphones). 


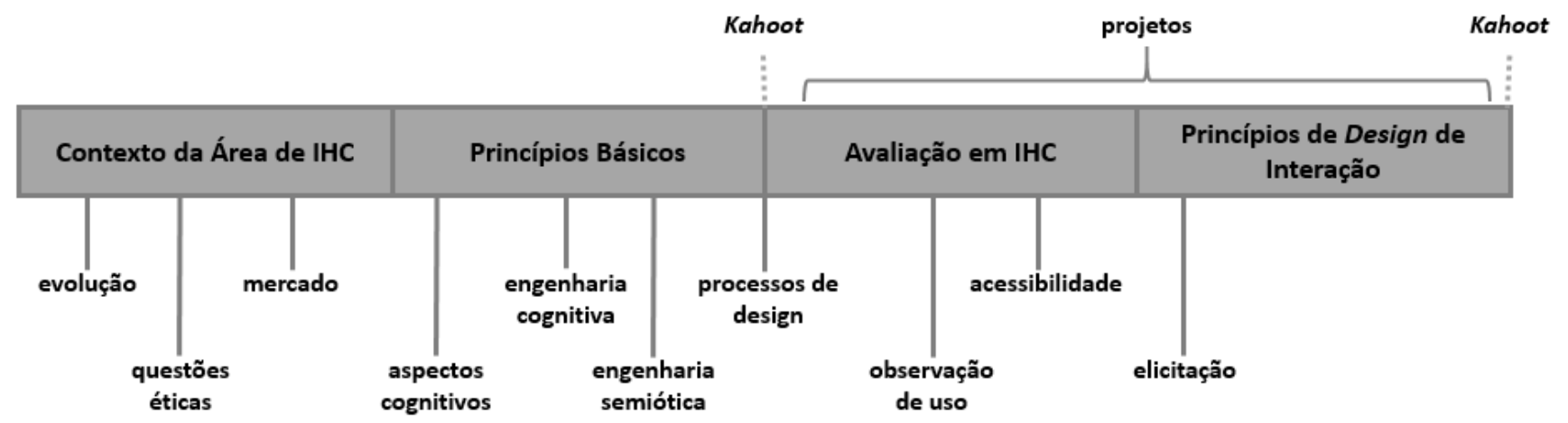

Figura 1: Exemplos de tópicos com atividades práticas relacionadas.

- Processos de design: ao final desta unidade (de Princípios Básicos), são trabalhados os diferentes ciclos de vida de IHC, no contexto do processo de design. Para refletir sobre a questão de iteratividade, característica principal do processo, primeiro é realizado uma dinâmica na qual os grupos devem construir um determinado artefato, e, a cada etapa, são recebidos novos requisitos, pela análise dos quais o artefato (construído com massinha de modelar) vai sendo refinado/modificado (um exemplo de requisitos e artefatos pode ser visto na Figura 2a). Além disto, os integrantes de cada grupo recebem papéis diferentes (como desenvolvedor, UX designer, etc) e algumas equipes tem um integrante com papel de usuário e outras não. Para a etapa final, as equipes recebem diferentes modelos de ciclo de vida e precisam analisalos, destacando seus pontos em comum. $\mathrm{O}$ foco principal de toda a atividade e a discussão da questão da iteratividade do processo e da importância da inclusão do usuário no mesmo.

\subsection{Atividades: Avaliação em IHC}

Durante a terceira unidade, referente a avaliação em IHC, destacam-se as seguintes atividades:

- Observação de uso: para trabalhar conceitos e métodos relacionados a observação de uso, a turma é dividida em grupos. Cada grupo, de posse de um terminado tópico (por exemplo: planejamento de um teste em laboratório, testes de usabilidade, avaliação de comunicabilidade, etc), deve criar um jogo de tabuleiro que explore os conceitos relacionados. Cada grupo recebe um "kit para criação" (exemplos de tabuleiros impressos, peões, dados, uma roleta e uma ampulheta), para servir de base ou de inspiração para sua construção, além dos slides de aula referentes ao tópico e de livros trazidos por eles e consultas via Internet. Eles têm o tempo de uma aula (2 períodos), e extra classe até a próxima, para a construção das regras do jogo e, na aula seguinte, a turma joga os diferentes jogos em formato de circuito/rodízio (exemplos podem ser vistos na Figura 2b).

- Acessibilidade: para trabalhar conceitos e métodos referentes a avaliação de acessibilidade é feita uma Gincana Virtual. Cada aluno, ao entrar na sala de aula, recebe um número, o qual representa sua equipe, e lhe é solicitado a ler as instruções da atividade na área Moodle da disciplina. Dentre as instruções, encontramse a forma de comunicação das equipes (apenas via sala de chat do Moodle identificada com o número de sua equipe) e as regras de postagem das respostas. Ao iniciar a aula, as tarefas são disponibilizadas e os alunos têm o tempo da aula ( 2 períodos) para se organizar, discutir as tarefas (via chat) e posta-las no Moodle. Destaca-se que todas as tarefas requerem um tempo de reflexão dos alunos, não sendo possível responder a maioria delas com apenas um "copia e cola" da Internet.

\subsection{Atividade: Princípios de Design de Interação}

Durante a última unidade, referente a princípios de design de interação, destaca-se a seguinte atividade:

- Elicitação: uma das técnicas de elicitação de dados trabalhadas é o card sorting (classificação de cartões). A aula referente a este tópico é dividida em três momentos: em um primeiro, se discute como funciona a técnica e suas possibilidades de aplicação; em um segundo momento, os alunos -divididos em gruposrecebem um conjunto de cartões contendo elementos lúdicos e necessitam classifica-los, de acordo com categorias criadas pelo grupo, e, depois, apresentar o resultado à turma, discutindo o porquê de sua categorização; por fim, os alunos recebem uma série de itens (originários de menus de um conhecido site de entretenimento), devendo, também, classifica-los, agora de acordo com as categorias preexistentes no referido site, e apresentar e refletir com os colegas, principalmente, as diferenças entre sua classificação e a classificação original. Na Figura $2 \mathrm{c}$ vemos exemplos das duas etapas de classificação de cartões. 


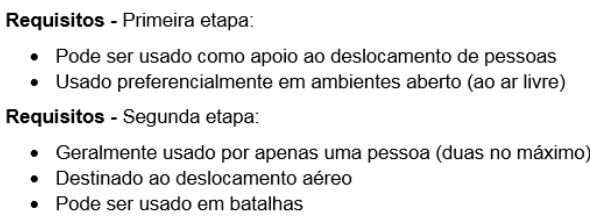

- Pode ser usado como apoio ao deslocamento de pessoas

- Usado preferencialmente em ambientes aberto (ao ar livre)

Requisitos - Segunda etapa:

- Geralmente usado por apenas uma pessoa (duas no máximo)

- Destinado ao deslocamento aéreo

- Pode ser usado em batalhas

\section{Requisitos}

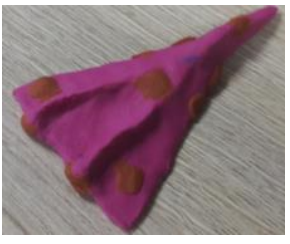

Exemplos de artefatos criados

(a)
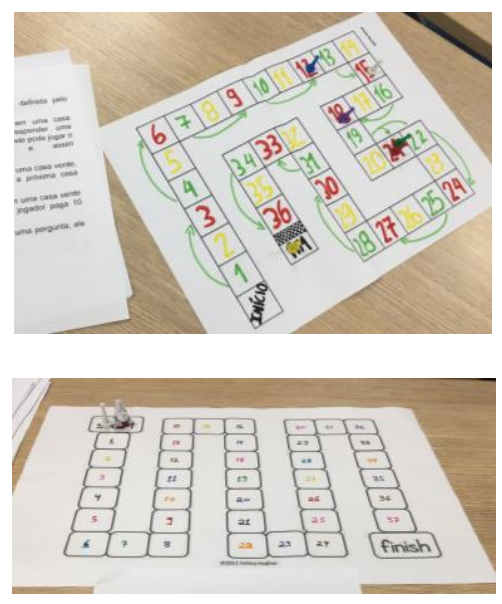

(b)

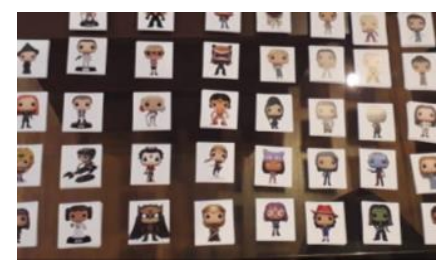

Classificação com elementos lúdicos

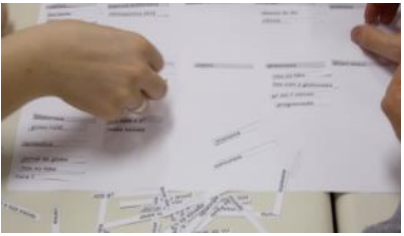

(re)classificação de itens de menu

(c)

Figura 2: Exemplos de atividades: (a) processo de design, (b) jogos de tabuleiro, (c) card sorting.

\section{CONSIDERAÇÕES FINAIS}

As diferentes atividades realizadas ao longo do semestre têm auxiliado a motivar os alunos a refletir mais durante as aulas, participando mais das mesmas. Competências como colaboração, solução de problemas, curiosidade, imaginação e pensamento crítico foram trabalhadas e o engajamento foi grande em várias atividades, principalmente as de mais "mãos na massa", como as apresentadas na Figura 2.

Destaca-se também que, além das atividades apresentadas nas subseções anteriores, outros exercícios também têm sido realizados, como as micro atividades de Pontual Falcão [9] e vários inspirados em um dos livros texto da disciplina [10]. Outro recurso que tem sido utilizado (ao final da segunda unidade e na aula anterior a prova) é a aplicação de um quiz, utilizando-se a ferramenta Kahoot ${ }^{1}$, atividade esta que é bastante apreciada pelos alunos.

As atividades e os exercícios apresentados têm sido realizados ao longo da disciplina, de acordo com o tópico sendo trabalhado, mas sem fazer parte do processo avaliativo formal. Para avaliação, além da prova final (há apenas uma prova compondo a avaliação), a disciplina conta com um conjunto de projetos. Estes projetos, relacionados às unidades de Avaliação e de Princípios de Design, têm sido aplicados -em sua grande maioria- alinhados às disciplinas de práticas de desenvolvimento de software, as quais atendem clientes reais. Desta forma, os alunos analisam casos reais, com a oportunidade de interagir com seus stakeholders, com sua equipe de projeto e desenvolvimento e, também, com seus futuros usuários, além de ter os resultados de seus trabalhos podendo ser usados como insumos para novas etapas de projeto e de desenvolvimento da disciplina parceira [11].
Por fim, destaca-se que, durante o semestre de 2019/1, ao longo do semestre, uma série de desafios foi lançada aos alunos, em uma abordagem de gamificação da sala de aula, estando estes resultados ainda em fase de análise.

\section{REFERÊNCIAS}

1. M.A. Amaral, S.A. Bim, C. Boscarioli e C. Maciel. 2015. Introducing Computer Science to Brazilian Girls in Elementary School Through HCI Concepts. Em: A. Marcus (eds) Design, User Experience, and Usability: Users and Interactions. DUXU 2015. Lecture Notes in Computer Science, vol 9187. Springer, Cham, Suíça.

2. L. Bacich e J. Moran. 2018. Metodologias ativas para uma educação inovadora: uma abordagem teórico-prática. Penso, Porto Alegre, RS, Brasil.

3. T. Bell, I.H. Witten e M. Fellows. 1999. Computer Science Unplugged: OffLine Activities and Games for All Ages (Original Book). http://csunplugged.org

4. T. Bell e J. Vahrenhold. 2018. CS Unplugged-How Is It Used, and Does It Work?. Em: HJ. Böckenhauer, D. Komm and W. Unger W. (eds) Adventures Between Lower Bounds and Higher Altitudes. Lecture Notes in Computer Science, vol 11011. Springer, Cham, Suíça.

5. F. Camargo e T. Daros. 2018. A sala de aula inovadora: estratégias pedagógicas para fomentar o aprendizado ativo. Penso, Porto Alegre, RS, Brasil.

6. A. Filatro e C.C. Cavalcanti. 2018. Metodologias Inov-ativas na educação presencial, a distância e corporativa. Saraiva Educação, São Paulo, SP, Brasil.

7. C. Maciel, S.A. Bim e C. Boscarioli. 2013. HCI with Chocolate: Introducing HCI Concepts to Brazilian Girls in Elementary School. In: Collazos C., Liborio A., Rusu C. (eds) Human Computer Interaction. CLIHC 2013. Lecture Notes in Computer Science, vol 8278. Springer, Cham, Suiça.

8. J. Mattar. 2017. Metodologias ativas: para educação presencial, blended e a distância. Artesanato Educacional, São Paulo, SP, Brasil.

9. T. Pontual Falcão. 2018. Miniatividades sobre conceitos básicos de IHC dinamizando aulas expositivas. Workshop sobre Educação em IHC, XVII Simpósio Brasileiro sobre Fatores Humanos em Sistemas Computacionais IHC 2018. SBC, Porto Alegre, RS, Brasil.

10. Y. Rogers, H. Sharp e J. Preece. Design de interação: além da interação humano-computador. Bookman, Porto Alegre, 2013. 599p.

11. M. Silveira e A.C.S. Dutra. Bringing Life to the Classroom: Engaging Students through the Integration of HCI in SE Projects. 21st International Conference on Enterprise Information Systems - ICEIS 2019. p. 390397.SCITEPRESS, Setúbal.

12. UC Computer Science. Education Unplugged: The show. Part 6: HCI - The Stroop effect. https://www.youtube.com/watch?v=4iHPgk2u9_s 